\title{
SPURIOUS OSCILLATIONS IN HIGH POWER KLYSTRONS*
}

\author{
B. Krietenstein, THD, Darmstadt, Germany, K. Ko, and T. Lee, \\ Stanford Linear Accelerator Center, Stanford University, Stanford, CA 94309 \\ U. Becker and T. Weiland, THD, Darmstadt, Germany \\ M. Dohlus, DESY, Hamburg, Germany
}

\section{- Abstract}

Spurious oscillations in high power klystrons are found to oceur in the gun region, in the cavities in the main body of the tube, or in the drift tunnel. The criteria that determine whether a mode will oseillate is that its beam loading be negative, and that the power it extracts from the beam exceeds its losses to external loading and wall dissipation. Using the electromagnetic and particle-in-cell modules of MAFIA, we bave devised oumerical techniques with which the quality factors $Q_{b}, Q_{c}$ and $Q_{0}$ can be evaluated and compared. Simulations involving a gun oseillation observed in the SLAC/DESY S-Band klystron will be reported.

\section{INTRODUCTION}

Oseillations at frequencies other than the operating fir quency are not uncommon in high power klystrons. The unwanted signals bave been detected in the pulse transformer tank in the gun region, and also in the input and output RF coupless. They occur without the RF drive sigDal, and have the signature of high- $Q$ resonances in that their frequencies are not affected by variation in operating parameters, such as the focussing magnetic field. S-Band klystrons built at SLAC bave experienced oscillations in the gun diode [1]), in the drift tunnel [2], and also in an output circuit of the double-gap type [3].

Spurious oscillations are undesirable since the electron beam couples to these modes as well as the desired signal frequency. If the amplitude of the spurious oseillation be comes very large the performance of the tube can be compromised. The main output signal may suffer from amplitude and phase instability which results in pulse shortening or decrease in efficiency. Thus, spurious oscillations can be a serious factor in limiting a klystron from reaching its designed performance if left unsuppressed. The identification and analysis of spurious modes are not easy to accomplish experimentally because the diagnostics are not set up to monitor such signals. Numerical modeling has proved to be valuable in microwave design and this paper describes the analytical efforts to address the spurious mode issue by computer simulation.

\section{COMPUTER ANALYSIS}

The ability of modern electromagnetic codes to study $R F$ modes in complex geometries, both in 2 and $3 D$, has enabled the klystron designer to identify spurious modes once their frequencies are known from measurements. Guided

\footnotetext{
- Work supported by the Department of Energy, contract DE-ACO3i6SFO0S15.
}

by the numerical solutions, he can then devise a method of suppression to be incorporated into the next prototype. Ideally, it would be more cost-effective to catch potentially unstable modes in the design stage before fabrieation and testing take place, thereby reducing the number of prototypes to be built. But this would require a complete analysis of the numerous modes that a kyystron circuit can support, which is a nontrivial task. The analysis would have to iselude not only the vacuum RF properties, but also the mode interaction with the electron beam under a range of operating conditions, in order to determine if any of them is unstable or not.

Beam-field interaction can be modeled by the ParticleIn-Cell (PIC) method, and 2D PIC codes such as CONDOR bave been used quite routinely in klystron tube design to optimize the extraction of power from the beam [4]. In this application, one is concerned only with the main signal which is an axisymmetric mode so that, except for the input and output $R F$ couplers, a $2 D(r, z)$ geometry suffices. Spurious oscillations, or the other hand, are not limited to monopole modes, but have been observed to be dipoie modes as well. Furthermore, external Joading by coupling waveguides now plays an important role. PIC modules are available in $3 D$ codes such as MAFIA, but their usuage in klystron design has been restricted, largely because of the substantial computer resources such simulations demand. In this work, we will deal with a spurious mode that is axisymmetric so that only $2 D$ caleulations are required. We first formulate the criteria that determine oscillation in a way that is computationally efficient. Instead of treating the problem in one simulation, we consider separately three competing effects: beam-field interation, external loading and wall dissipation. We use the electromagnetic/PIC modules of MAFIA [5] to ana]yse a gun oscillation problem and dernonstrate the method with which to evaluate each effect. We next present the numerical results and discuss the eficacy of the approach.

\section{CRITERIA FOR OSCILLATION}

There are two criteria that determine whether a mode can be driven to oscillate by a beam. The first and necessary condition is that the beam tranfers power to the mode. The second condition is that the power lost by the mode to external loading and wall dissipation is less than the power obtained from the beam. As a result, there is a net gain in mode energy and the oscillation grows. Computationally, it is prohibitively expensive to model this power balance process. To begin with, one needs a disproportionately small grid step to resolve the skin depth due to finite wall conductivity. It also takes an unrealistic number of 
particles to maintain an electron beam flow over the losg growth time of the instability.

Alternatively, one argues that up to the instability, threshold each power transfer acts independently, so the:fore they can be treated separately. We define the to:al quality factor $Q_{\text {tot }}$ of a mode as:

$$
1 / Q_{t a t}=1 / Q_{b}+1 / Q_{c}+1 / Q_{b},
$$

where $Q_{0}, Q_{e}$ and $Q_{b}$ are the wall loss $Q$, the ezternal $Q$ and the beam-loaded $Q$ respectively. If we express $Q$ is.

$$
Q=\omega U / P
$$

where $w$ is the angular frequency of the mode, $U$ is the timeaveraged stored energy and $P$ is the power transferred, then Eq. (1) describes the power exchange between the mode and the beam, and the power loss by the mode to the cirevit environment. If we take power gain as negative, it follons from Eq. (1) that the oscillation criterion is

$$
Q_{\text {tot }}<0 \text {. }
$$

Since $Q_{0}$ and $Q_{\text {a }}$ are alway's positive, then the sign and value of $Q_{b}$ will determine unstable resonant modes. A necessary step before one can calculate the quality facto:s is the identification of the spurious mode, and this we will takie up next.

\section{MODE IDENTIFICATION}

One outstanding feature of spurious modes is their lo calization within some region of the klystron to form a resonant eirevit. The most efficient way to search for resonances is with eigenmode solvers. Among the many solutions such solvers generate, one looks for modes in the proper frequency range that bave diminishing fields to wards the enclosing boundaries. Non-localized modes rill form standing waves between opposing boundaries while localized modes will have exponentially decaying fields. A direct consequence is that localized modes will experience selatively small frequency shifts when different field conditions (eleetric wall : $E_{\text {iangential }}=0$ or magnetic wall : $\left.B_{\text {iengential }}=0\right)$ are imposed at the bouncaries.

During the testing of the $150 \mathrm{MW}$ S-band klystron that SLAC is building for DESY, a gun oscillation was discovered when a stable $1.365 \mathrm{GHz}$ signal was detected at the sight window of the pulse transformer tank [1] (with no RF signal applied). Fig. 1 shows a MAFIA calculation of a mode at the same frequency that is localized inside the focus electrode. A small gap connects this small cavity to the main body of the gun diode. By comparison, Fig. 2 shows a nearby mode at $1.277 \mathrm{GHz}$ that oecupies the en-, tire diode. Changing the top boundary from an electric to a magnetic wall results in a frequency shift and a different mode pattern for this mode. In contrast, the localized mode is relatively unchanged, indicating the presence of a high- $Q$ resonant structure. We conclude that it is a likely candidale responsible for the spurious oscillation and proceed to determine its $Q$ factors.

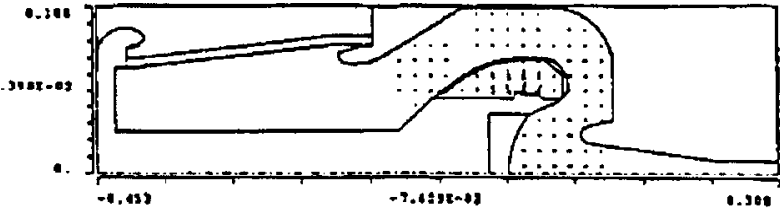

Figure 1. $1.365 \mathrm{GEz}$ mode in the SLAC/DESY gun diode.

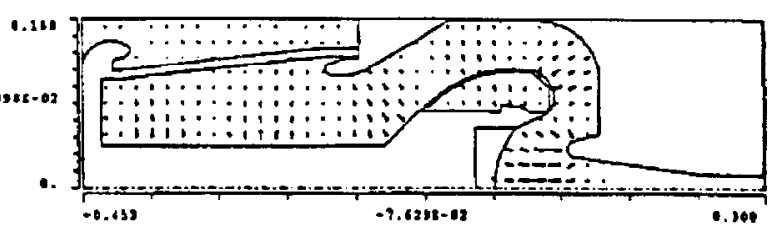

Figure 2. $1.277 \mathrm{GHz}$ mode in the same geometry.

\section{Q DETERMINATION}

\section{Wall Loss-Q.}

Once a likely resonant mode is indentified from the eigenmode spectrum, it is straightforward to calculate its Qo by standard perturbation metbod. Most field solvers, including MAFIA, provide this result automatically via postprocessing. The time-average porer absorbed per urit area at the wall is given by

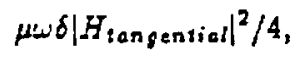

where $\delta$ is the skjp depth $(2 / \mu \omega \kappa)^{(1 / 2)}$ and $\kappa$ the finite conductivity of the wall material. From Eq. (2), then

$$
Q_{0}=\omega U / P_{w} \text {, }
$$

where $P_{w}$ is the integral of $E q$. (4) over the wall surface. $Q$. due to copper loss typically ranges from several to ters of thousands at RF frequencies, and is not a major factor in determining $Q_{T}$. However, in situations where external loading is ineffective or not applicable, lossy wall material (e.g. stainless steel) is sometimes introduced in areas of high surface currents to provide additional Ohmic loss for mode suppression.

\section{External Loading-Q.}

Or the various methods to determine the external $Q$ of a mode, we will choose the one which is the most direct. We load the eigenmode from the previous calculation as the initial conditjons to a time-domain simulation. As opposed to the eigenmode analysis which assumes a closed cavity, power flow across the boundaries are now permissable via ports that are terminated in matched loads. In the gua-diode of Fig. 1, one port is the outer boundary of the pulse tranformer tank at top left, while the other is at the entry into the drift tunnel to the bottom right. With the ports open, the initially confined electromagnetic fields can radiate through these apertures and decay. After the initial transient has subsided, one can calculate the decay time constant $T_{e}$ from the field values at successive time inte:vals. The external loading effect is then determined directly from the relation

$$
Q_{e}=\omega \tau_{c} / 2
$$




\section{Beam Looding- $Q_{3}$}

The beam loading of a mode comes about because the mode induces bunching in the beam which then interac:s with the mode field to produce energy exchange. In the abseace of losses, we can use exergy conservation and equate the rate of change of stored energy to the power transfer between the mode and the beam. We arrive at an expression for $Q_{b}$ similar to Eq. (2), namely,

$$
Q_{b}=\omega U / P_{b} \text {. }
$$

The time-average power flow $P_{t}$ is given by the integ:al over the beam volume

$$
P_{b}=\frac{1}{T} \int_{i}^{t+T} \int_{V} \overrightarrow{J_{m}} \cdot \overrightarrow{E_{m}} d V d t
$$

where $J_{m}$ is the induced current density, $E_{m}$ is the electric field of the mode, and $T$ its period. The facility to evalua:e $P_{b}$ is currently under construction in MAFIA, but we can alternatively find $Q$, from the time constant the same way it is done in determining $Q_{e}$.

\section{GUN OSCILLATION SIMULATION}

As roted before, the computation of $Q_{b}$ requires a full PIC simulation that includes relativistic and space charge effects [6]. The MAFIA PIC moduie bas this capability so we use it to model the gun oscillation problem. The MAFIA simulation consists of a de beam, a static field between cathode and anode, a focussing magnetic field and the RF mode under consideration. The injection parameters for the beam at the cathode are taken from previous EGUN [7] results, while the MAFIA static module provides the gradient $E_{8}$ and the magnetic field $B_{0}$. The RF mode $E_{m}$ is taken from the eigenmode calculation as before.

Without the RF mode, the PIC module essentially if produces the beam optics results from EGUN. The RF mode is then added with its amplitude scaled to a small fraction of the static field amplitude. This way the RF mode serves as a test field that modulates the lowest order beam equilibrium. Fig. 3 shows a time snapshot of the simulation. The beam is constituted of fifteen rays and the field ratio used is $E_{m} / E_{s}=0.1$. Note that the gun diode is much smaller than previously considered. We provide the justification for the reduced geometry below.

In the absence of external loading and $x^{\prime}$ all loss, the RF mode is basically a standing wave resonance. A resonant circuit is essentially unebanged if shorted properly at one of its node points. The region from the focus electrode to the pulse transformer tank does not play a part in beamloading except for the energy stored there. Fig. 3 show's. the placement of a shorting plane which removes this paft of the circuit but leaves the frequency and field pattern of the RF mode unchanged. The geometry is now smaller, and because the total stored energy is less, any time constant related to it also is faster, both of which is good for simulation. However, one needs to scale by the proper ratio of the stored energy in the full geometry to that in the reduced one to obtain the physically correct values.

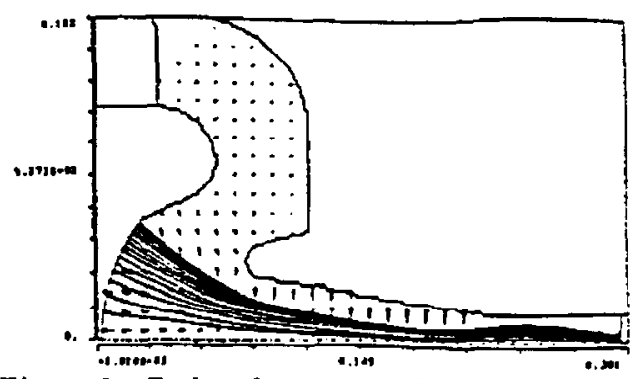

Figure 3. Reduced geometry for the PIC simulation.

VII. NUMERICAL RESUITS

The calculations from the previous sections on the localised $1.365 \mathrm{GHz}$ mode are summarized in Table 1. For comparison, the results on the $1.277 \mathrm{GHz}$ mode are also given. As expected, the $Q_{0}$ 's do not play a role. The $1.277 \mathrm{GHz}$ signal has negative beam loading, but is negligible when compared with the heavy external loading, so the net result for $Q_{10}$ is positive. On the other hand, the $1.365 \mathrm{GEz}$ signal couples so weakly to external loads that the stronger negative beam loading leads to a negative $Q_{\text {tot }}$, and results in oscillation. After the gap at the focus electrode in the SLAC/DESY klystron gun was shorted to eliminate the $1.365 \mathrm{GHz}$ unstable mode, the klystion was processed up to full power without experiencing the previously observed gun oscillation.

\begin{tabular}{|c|c|c|c|c|}
\hline $\begin{array}{c}\text { Frequency } \\
{[G \mathrm{~Hz}]}\end{array}$ & $Q_{0}$ & $Q_{e}$ & $Q_{t}$ & $Q_{10:}$ \\
\hline 1.257 & 25500 & 45 & -1340 & 46 \\
\hline 1.365 & 13300 & 5000 & -728 & -910 \\
\hline
\end{tabular}

Table 1. Calculated $Q$ 's for the 1.365 and $1.277 \mathrm{GEz}$ signal. VIII. SUMMARY

We have presented an analytical method to study spurious oscillation by computer simulation, and have applied it to gun oscillation problem with encouraging results. Further generalization of this approach to other klystron oscillations, including those in which $3 D$ effects can be important, is being considered.

\section{Acknowledgements}

We thank D. Sprehn for useful discussions. One of the authors (B. Krieterstein) ajso achowowledges R. Ruth for his generous hospiiality and support during his stay with the ATSP Dept. at SLAC.

\section{References}

(1) D. Sprehn, R.M. Philips, and G. Caryotakis, The Design and Peformasce of 150-MW S-Band Klyctrons, SIAC PUB 6677, Staniord Linear Accelerator Center, Sep. 1994.

[2] K. Ko, Private commurication.

[3] K. Ko el, d., Linear Collider RF Structure Design Using ARGUS, Proceedings of the First Energy Research Power Supereomputen Users Sympotium, Gaithersburz, MD 1991.

[4] K.R. Eppley, Modelling RF Sources Using 2-D PIC CODES, AIP Conference Proceedings on Computational Accelerabor Physics, Los Nlamos, NM 1993.

[5] The MAFIA collaboration, Ustr', Guide MAFIA Vetsion s.z, CST GmbH, Lauteschlagentr.38, D6100 Darmstadt.

[6] B. Krietenstein, Simulation of Oscillations in High Vollage Klystrons with MAFIA, Institul '"ur Hochlrequenztechnik, Technische Hochsechule Darmstadt, Juli 1994.

[7] W.B. Hermarnsfejdt, Electron tra jectory program, SLAC PUB 226, Stanford Lineas Acceleralor Center, Nov. 1979. 